\title{
A Case of COVID-19 - Tianjin Municipality, China, November 7, 2020
}

\author{
COVID-19 Field Response Group ${ }^{1}$; Laboratory Testing Group ${ }^{1}$; Yang Song'; Xiang Zhao ${ }^{2}$; Xiaoyan $\mathrm{Li}^{1, *, *}$; Wenbo $\mathrm{Xu}^{2, *}$
}

On November 7, 2020, Tianjin Municipality received notification from Dezhou City, Shandong Province that specimens of the outer packaging of imported frozen pork products shipped from Germany via Tianjin Port were positive for coronavirus disease 2019 (COVID-19) viral nucleic acid. Tianjin Municipal Government then conducted an investigation for people working at the Tianjin Hailian Frozen Food Co., Ltd. On November 8, a 38-year-old cold chain dockworker from the company was found positive via nucleic acid test. The patient had participated in the transport of the infected batch of frozen products on November 4. The patient was transferred to a designated hospital for medical isolation and was classified as a standard clinical case of COVID-19. As of November 11, 8 close contacts of the patient and 13 employees of the company were tested 2 times with nucleic acid and antibody tests and all returned negative results. In addition, 30 samples of items suspected of contamination and other environmental samples at the company were collected, of which 1 sample of the door handle of the cold storage room was found to be positive. Nucleic acid tests for 156 people in the patient's residential building were all negative, and the scope of testing was further expanded. Nucleic acid tests were performed on 2,186 people from 765 households in 25 buildings in the patient's residential area, and all results were negative.

On November 8, full-length genome sequencing of a specimen collected from the Tianjin COVID-19 patient was performed on the Illumina MiniSeq platform. Compared with the Wuhan reference sequence (EPI_ISL_402119)(1), a total of 9 nucleotide mutation sites were detected in the Tianjin strain, among which 6 (C241T, C1059T, C3037T, C14408T, A23403G, and G25563T) were detected as characteristic of L-lineage European branch II.1/lineage B.1(2)(Figure 1). In addition, the Tianjin strain had 3 unique nucleotide mutation sites (C601A, $\mathrm{C} 4965 \mathrm{~T}$, and $\mathrm{C} 26625 \mathrm{~T}$ ) and no sequence with more than 2 of these nucleotide mutation sites was retrieved in GISAID and Genbank database.

In addition, 2 Canadian strains (GISAID IDs:
EPI_ISL_586371 and EPI_ISL_469240) circulating in March had 7 nucleotide mutation sites, and all 7 sites also appeared in the Tianjin strain, which included the 6 sites that defined European family of the L genotype II.1 and a unique site of C4965T or C26625T. Though the Tianjin strain was genetically closely related to these 2 Canadian strains, the similar viruses might have circulated in many countries.

Compared with recent strains spreading worldwide (with around 15 nucleotide mutation sites from the Wuhan reference strain), the number of mutation sites of the Tianjin strains was relatively low, which indicated that the virus may have been transmitting undetected through contamination of the cold chain product that may have occurred months ago and retained its infectivity.

From November 9 to 11, the Tianjijn Municipal Government completed comprehensive nucleic acid testing for imported frozen goods and all persons with contact. The municipal government also closed facilities found with abnormal frozen goods and isolated all suspected products.

Funding: This work was supported by Tianjin Municipality Science and Technology Project of the Tianjin National Health Commission (2020XKM01, 2020XKZ01).

doi: $10.46234 / \mathrm{ccdcw} 2020.241$

\# Corresponding authors: Xiaoyan Li, xiaoyanli1291@163.com; Wenbo Xu, xuwb@ivdc.chinacdc.cn.

\footnotetext{
${ }^{1}$ Tianjin Center for Disease Control and Prevention, Tianjin, China; ${ }^{2}$ National Institute for Viral Disease Control and Prevention, China CDC, Beijing, China.
}

Submitted: November 11, 2020; Accepted: November 12, 2020

\section{REFERENCES}

1. Wu F, Zhao S, Yu B, Chen YM, Wang W, Song ZG, et al. A new coronavirus associated with human respiratory disease in China. Nature 2020;579(7798):265 - 9. http://dx.doi.org/10.1038/s41586-020-2008-3.

2. Rambaut A, Holmes EC, O’Toole Á, Hill V, McCrone JT, Ruis C, et al. A dynamic nomenclature proposal for SARS-CoV-2 lineages to assist genomic epidemiology. Nat Microbiol 2020. http://dx.doi.org/10.1038/ s41564-020-0770-5. 


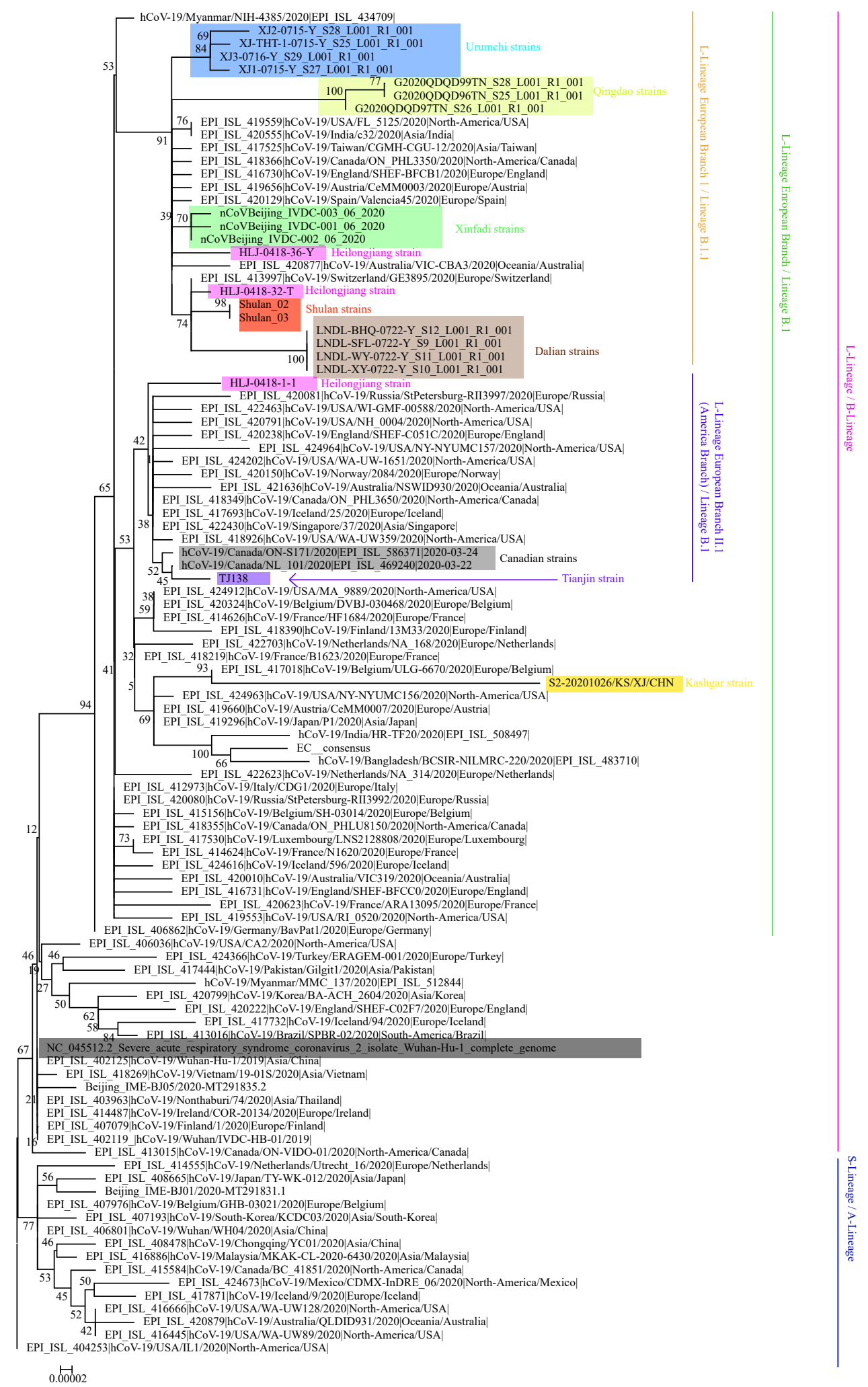

FIGURE 1. Phylogenetic tree based on the full-length genome sequences of the COVID-19 virus. The strains associated with specific outbreaks are as follows: Tianjin Municipality (purple); Kashgar Prefecture (yellow); Urumchi City (blue); Wuhan City in December 2019 (dark gray); Beijing Municipality Xinfadi Wholesale Market (green); northeastern China including Shulan City and Heilongjiang Province related to imported cases (orange and pink, respectively); and Dalian City (brown). The Canadian strains that had high genomic homology with the Tianjin strain were marked in light gray. The $S(A)-$ or $L(B)-$ lineage and sublineages of the COVID-19 virus were marked and colored on the right. 